\title{
Minimal Affine Boundaries of Convex Sets
}

\author{
Toma TONEV* \\ Bulgarian Academy of Sciences \\ (Communicated by S. Suzuki)
}

\section{Introduction.}

Let $M$ be a compact convex set in a real locally convex linear topological space $V$ and denote by $A(M)$ the set of restrictions on $M$ of all real affine and continuous functionals in $V$, i.e. $f \in A(M)$ iff $f(t x+(1-t) y)=$ $t f(x)+(1-t) f(y)$ for any $t \in R$. Remind that a subset $N$ of $M$ is called an end subset of $M$ iff it consists of points $z$ that satisfy the following condition: $z$ can not be represented as $z=\lambda x+\mu y$ with $\lambda>0, \mu>0, \lambda+\mu=1$, unless $x$ and $y$ belong to $N$. Extreme points of $M$ are the points that are end subsets of $M$. Let $E(M)$ stand for the closure of extreme points of $M$. This is the smallest closed subset of $M$ within which any positive element of $A(M)$ attains its minimum. Indeed, let $f \in A(M)$, $f>0$, and let $\min _{x \in M} f(x)=a<b=\min _{x \in E(M)} f(x)$. Since $f$ is affine, the set $M \cap\{f(x) \geqq b\}$ is a compact convex set that contains $E(M)$ and consequently it contains also the closed convex hull of $E(M)$, i.e., it contains the whole set $M$ according to the Krein-Milman's theorem (e.g. [1]). Hence $f(x) \geqq b>a$ on $M$, that is a contradiction. So every positive element of $A(M)$ attains its minimum within $E(M)$. If a closed subset $N$ of $M$ possesses the same property, then its closed convex hull $[\langle N\rangle]$ will coincide with $M$. In fact, if $[\langle N\rangle] \neq M$ we can find a positive continuous affine functional $f \in A(M)$ for which $f(x) \geqq a>0$ on $[\langle N\rangle]$ but $f\left(x_{0}\right)<a$ for some point $x_{0} \in M$ in contradiction with our supposition on $N$. But the equality $[\langle N\rangle]=M$ implies that $N \supset E(M)$ since the latter is the smallest closed subset of $M$ for which $[\langle N\rangle]=M$ (e.g. [2]). Here we introduce $n$-dimensional analogues to the closure $E(M)$ of extreme points of a compact convex set $M$.

§ 1. Affine $n$-boundaries.

Denote by $A^{n}(M)$ the set of all $n$-tuples $\left(f_{1}, \cdots, f_{n}\right)$ of elements of Received July 15, 1987

* Research partially supported by Grant Nr 386 from the Committee of Science (Bulgaria). 
$A(M)$, by $Z\left(f_{1}, \cdots, f_{n}\right)$ the zero set of $\left(f_{1}, \cdots, f_{n}\right)$, i.e. $Z\left(f_{1}, \cdots, f_{n}\right)=$ $\left\{x \in M: f_{1}(x)=f_{2}(x)=\cdots=f_{n}(x)=0\right\}$ and by $A_{*}^{n}(M)$ the set of all regular $n$ tuples over $A(M)$, i.e. $\left(f_{1}, \cdots, f_{n}\right) \in A_{*}^{n}(M)$ iff $Z\left(f_{1}, \cdots, f_{n}\right)=\varnothing . \quad A_{*}^{0}(M)$ will stand for all constant elements of $A(M)$. Let $\left\|\left(f_{1}, \cdots, f_{n}\right)\right\|$ be the following function on $M$ :

$$
\left\|\left(f_{1}, \cdots, f_{n}\right)(x)\right\|=\left(\sum_{j=1}^{n} f_{j}^{2}(x)\right)^{1 / 2} .
$$

DEFinition 1. A subset $E$ of a compact convex subset $M$ of a real locally convex linear topological space $V$ is called an affine $n$-boundary of $M$ iff for every regular $n$-tuple $\left(f_{1}, \cdots, f_{n}\right)$ of affine continuous functionals on $M$ there exists a point $x_{0}$ belonging to $E$ such that for any $x \in M$ it holds:

$$
\left\|\left(f_{1}, \cdots, f_{n}\right)\left(x_{0}\right)\right\| \leqq\left\|\left(f_{1}, \cdots, f_{n}\right)(x)\right\|,
$$

i.e. iff the minimum of the function $\left\|\left(f_{1}, \cdots, f_{n}\right)\right\|$ is attained within $E$ for every regular $n$-tuple $\left(f_{1}, \cdots, f_{n}\right) \in A_{*}^{n}(M)$.

DEFinition 2. The intersection $E_{n}(M)$ of all closed affine $n$-boundaries of a compact convex subset $M$ of $V$ is called the minimal affine $n$-boundary of $M$.

It is clear that $E_{1}(M) \subset E_{2}(M) \subset \cdots \subset E_{n}(M) \subset \cdots$. According to the remark from the Introduction, we have that $E_{1}(M)=E(M) \neq \varnothing$. The next theorem shows that minimal affine $n$-boundaries of $M$ are nonempty subsets of $M$ for every $n>1$ and, moreover, it gives a description of them.

TheOREM 1. The sets

$$
\left[\cup\left\{E\left(Z\left(f_{1}, \cdots, f_{n-1}\right)\right):\left(f_{1}, \cdots, f_{n-1}\right) \in A^{n-1}(M)\right\}\right]
$$

coincide with the minimal affine $n$-boundaries $E_{n}(M)$ of compact convex subsets $M$ of $V$, where $[N]$ denotes the closure of $N$ for a subset $N$ in $V$.

Proof. First we shall prove that the set (3) is an affine $n$-boundary of $M$. Let $\left(f_{1}, \cdots, f_{n}\right) \in A_{*}^{n}(M)$ and $x_{0} \in M$. Without loss of generality (applying, if necessary, certain orthogonal transformation in $\boldsymbol{R}^{n}$ ) we can assume that $f_{j}\left(x_{0}\right)=0$ for any $j>1$, so that $\left(f_{1}\left(x_{0}\right), f_{2}\left(x_{0}\right), \cdots, f_{n}\left(x_{0}\right)\right)=$ $\left(f_{1}\left(x_{0}\right), 0, \cdots, 0\right)$ and $\left\|\left(f_{1}, \cdots, f_{n}\right)\left(x_{0}\right)\right\|^{2}=f_{1}^{2}\left(x_{0}\right)$. The set $Z_{1}=Z\left(f_{2}, \cdots, f_{n}\right)$ is an affine manifold, i.e. a translated linear subspace of $V$. Because $f_{1}$ does not vanish at $Z_{1}$ and $x_{0} \in M \cap Z_{1}, f_{1}^{2}\left(x_{0}\right) \geqq \min _{E\left(Z_{1}\right)} f_{1}^{2}(x)$ according to our remark in the Introduction, applied to $Z_{1}$ and $\left.f_{1}\right|_{Z_{1}}$. Consequently 


$$
\begin{aligned}
& \left\|\left(f_{1}, \cdots, f_{n}\right)\left(x_{0}\right)\right\|^{2}=\sum_{j=1}^{n} f_{j}^{2}\left(x_{0}\right)=f_{1}^{2}\left(x_{0}\right) \geqq \min _{E\left(Z_{1}\right)} f_{1}^{2}(x)=\min _{E\left(Z_{1}\right)}\left(\sum_{j=1}^{n} f_{j}^{2}(x)\right) \\
& \quad \geqq \inf \left\{\sum_{j=1}^{n} f_{j}^{2}(x): x \in \cup\left\{E\left(Z\left(g_{1}, \cdots, g_{n-1}\right)\right):\left(g_{1}, \cdots, g_{n-1}\right) \in A^{n-1}(M)\right\}\right\} .
\end{aligned}
$$

Hence the continuous function $\left\|\left(f_{1}, \cdots, f_{n}\right)(x)\right\|$ attains its minimum within the set $\left[\cup\left\{E\left(Z\left(f_{1}, \cdots, f_{n-1}\right):\left(f_{1}, \cdots, f_{n-1}\right) \in A^{n-1}(M)\right\}\right]\right.$ for any regular $n$ tuple $\left(f_{1}, \cdots, f_{n}\right) \in A_{*}^{n}(M)$, i.e. (3) is an affine boundary of $M$. But (3) is the smallest affine $n$-boundary of $M$. Indeed, let $E \subset M$ be a closed affine $n$-boundary of $M$, i.e. let the minimum of the function $\left\|\left(f_{1}, \cdots, f_{n}\right)(x)\right\|$ is attained within $E$ for any regular $n$-tuple over $A(M)$. Let $\left(g_{1}, \cdots, g_{n-1}\right)$ be a fixed $(n-1)$-tuple over $A(M)$ and suppose that for some $f \in A(M)$ the restriction $\left.f\right|_{Z\left(g_{1}, \cdots, g_{n-1}\right)}$ is positive on the set $Z\left(g_{1}, \cdots, g_{n-1}\right)$ and that $f(x) \geqq r>0$ for some positive $r$ and for every $x \in Z\left(g_{1}, \cdots, g_{n-1}\right) \cap E$. We shall show that then $f(x) \geqq r$ on the whole $Z\left(g_{1}, \cdots, g_{n-1}\right)$. For any $\varepsilon>0$, $\varepsilon<r$ there exists a neighborhood $U_{\varepsilon} \subset M$ of the set $Z\left(g_{1}, \cdots, g_{n-1}\right) \cap E$ on which $f(x) \geqq r-\varepsilon$. Consequently for some positive constant $C_{\varepsilon}$, big enough, on $E$ we will have:

$$
C_{\varepsilon}^{2} \sum_{j=1}^{n-1} g_{j}^{2}(x)+f^{2}(x) \geqq(r-\varepsilon)^{2}
$$

Consequently (4) will hold on the whole $M$ because the $n$-tuple $\left(C_{\varepsilon} g_{1}, \cdots\right.$, $\left.C_{\varepsilon} g_{n-1}, f\right)$ is regular and $E$ is a closed affine $n$-boundary of $M$. In particular on $Z\left(g_{1}, \cdots, g_{n-1}\right)$ we will have that $f^{2}(x) \geqq(r-\varepsilon)^{2}$, from where $f^{2}(x) \geqq r^{2}$ because of the liberty of the choice of $\varepsilon$. We obtain that all affine functionals of $A\left(Z\left(g_{1}, \cdots, g_{n-1}\right)\right)$ that are positive attain their minimums within $Z\left(g_{1}, \cdots, g_{n-1}\right) \cap E$, wherefrom $Z\left(g_{1}, \cdots, g_{n-1}\right) \cap E \supset E\left(Z\left(g_{1}, \cdots\right.\right.$, $\left.g_{n-1}\right)$ ) because the latter set is the smallest closed affine 1-boundary of $Z\left(g_{1}, \cdots, g_{n-1}\right)$. Now $E \supset \cup\left\{Z\left(g_{1}, \cdots, g_{n-1}\right) \cap E:\left(g_{1}, \cdots, g_{n-1}\right) \in A^{n-1}(M)\right\} \supset$ $\cup\left\{E\left(Z\left(g_{1}, \cdots, g_{n-1}\right)\right):\left(g_{1}, \cdots, g_{n-1}\right) \in A^{n-1}(M)\right\}$ and by taking the closures we obtain finally that $E$ contains the set (3).

Q.E.D.

\section{$\S 2$. Properties of $\boldsymbol{n}$-affine boundaries.}

COROLlary 1. The range of the minimal affine n-boundary of a compact convex subset $M$ of $V$ through any $n$-tuple $\left(f_{1}, \cdots, f_{n}\right)$ of affine functionals from $A(M)$ contains the topological boundary of the range of $M$, i.e.

$$
\left(f_{1}, \cdots, f_{n}\right)\left(E_{n}(M)\right) \supset b\left(\left(f_{1}, \cdots, f_{n}\right)(M)\right), \quad \forall\left(f_{1}, \cdots, f_{n}\right) \in A^{n}(M) .
$$

Proof. Supposing that $b\left(\left(f_{1}, \cdots, f_{n}\right)(M)\right) \backslash\left(f_{1}, \cdots, f_{n}\right)\left(E_{n}(M)\right) \neq \varnothing$, let 
$x_{0}$ be such a point of $M$ that $\left(f_{1}, \cdots, f_{n}\right)\left(x_{0}\right) \in b\left(\left(f_{1}, \cdots, f_{n}\right)(M)\right) \backslash\left(f_{1}, \cdots\right.$, $\left.f_{n}\right)\left(E_{n}(M)\right)$ and let

$$
\min _{E_{n}(\boldsymbol{M})}\left\|\left(f_{1}, \cdots, f_{n}\right)\left(x_{0}\right)-\left(f_{1}, \cdots, f_{n}\right)(x)\right\|=\delta>0 .
$$

The continuous function $\left\|\left(f_{1}, \cdots, f_{n}\right)(x)-X^{0}\right\|$ on $M$, where $X^{0}=\left(x_{1}^{0}, \cdots, x_{n}^{0}\right)$ is a fixed point from $R^{n} \backslash\left(f_{1}, \cdots, f_{n}\right)(M)$ with $\left\|X^{0}-\left(f_{1}, \cdots, f_{n}\right)\left(x_{0}\right)\right\|<\delta / 2$, satisfies the following inequality:

$$
\begin{aligned}
& \left\|\left(f_{1}-x_{1}^{0}, \cdots, f_{n}-x_{n}^{0}\right)(x)\right\|=\left\|\left(f_{1}, \cdots, f_{n}\right)(x)-X^{0}\right\| \\
& \quad \geqq\left\|\left(f_{1}, \cdots, f_{n}\right)(x)-\left(f_{1}, \cdots, f_{n}\right)\left(x_{0}\right)\right\|-\left\|\left(f_{1}, \cdots, f_{n}\right)\left(x_{0}\right)-X^{0}\right\| \mid \geqq \delta / 2
\end{aligned}
$$

for any $x \in E_{n}(M)$. Hence

$$
\left\|\left(f_{1}-x_{1}^{0}, \cdots, f_{n}-x_{n}^{0}\right)(x)\right\|=\left\|\left(f_{1}, \cdots, f_{n}\right)(x)-X^{0}\right\| \geqq \delta / 2
$$

for any $x \in M$ because $\left(f_{1}-x_{1}^{0}, \cdots, f_{n}-x_{n}^{0}\right)$ is a regular $n$-tuple over $A(M)$ (since $\left.X^{0} \notin\left(f_{1}, \cdots, f_{n}\right)(M)\right)$ in contradiction with the choice of $X^{0}$. Consequently $b\left(\left(f_{1}, \cdots, f_{n}\right)(M)\right) \backslash\left(f_{1}, \cdots, f_{n}\right)\left(E_{n}(M)\right)=\varnothing$.

Q.E.D.

THEOREM 2. The minimal affine n-boundary of a compact convex subset $M$ of $V$ coincides with the intersection of all closed subsets $E$ of $M$, such that $\left(f_{1}, \cdots, f_{n}\right)(E) \supset b\left(\left(f_{1}, \cdots, f_{n}\right)(M)\right)$ for every $n$-tuple $\left(f_{1}, \cdots\right.$, $\left.f_{n}\right) \in A^{n}(M)$, i.e.

$$
\begin{array}{r}
E_{n}(M)=\cap\left\{E: E=[E] \subset M,\left(f_{1}, \cdots, f_{n}\right)(E) \supset b\left(\left(f_{1}, \cdots, f_{n}\right)(M)\right)\right. \\
\left.\quad \text { for each }\left(f_{1}, \cdots, f_{n}\right) \in A^{n}(M)\right\} .
\end{array}
$$

Proof. Corollary 1 shows that $E_{n}(M)$ contains the right hand side set of (6). Let $E$ be a closed subset of $M$ such that $\left(f_{1}, \cdots, f_{n}\right)(E) \supset$ $b\left(\left(f_{1}, \cdots, f_{n}\right)(M)\right)$ for every $n$-tuple $\left(f_{1}, \cdots, f_{n}\right) \in A^{n}(M)$ and let $\left(g_{1}, \cdots, g_{n}\right)$ be a fixed regular $n$-tuple over $A(M)$. Because of $\left(g_{1}, \cdots, g_{n}\right)(M) \nexists \nexists$ $(0, \cdots, 0)$, we can find a point $X^{0} \in b\left(\left(g_{1}, \cdots, g_{n}\right)(M)\right)$ such that $\left\|X^{0}\right\|=$ $\left\|\left(x_{1}^{0}, \cdots, x_{n}^{0}\right)\right\|=\min _{x \in M}\left\|\left(g_{1}, \cdots, g_{n}\right)(x)\right\|$. Now

$$
\begin{aligned}
\left\|\left(g_{1}, \cdots, g_{n}\right)(x)\right\| \geqq\left\|X^{0}\right\|=\min _{x \in M}\left\|\left(g_{1}, \cdots, g_{n}\right)(x)\right\|=\min _{X \in\left(g_{1}, \cdots, g_{n}\right)(u)}\|X\| \\
\quad=\min _{X \in b\left(\left(g_{1}, \cdots, g_{n}\right)(M)\right)}\|X\| \geqq \min _{X \in\left(g_{1}, \cdots, g_{n}\right)(E)}\|X\|=\min _{x \in E}\left\|\left(g_{1}, \cdots, g_{n}\right)(x)\right\|,
\end{aligned}
$$

because $\left(g_{1}, \cdots, g_{n}\right)(E) \supset b\left(\left(g_{1}, \cdots, g_{n}\right)(M)\right)$ according to our supposition. Consequently the minimum of the function $\left\|\left(f_{1}, \cdots, f_{n}\right)(x)\right\|$ is attained within $E$ for every regular $n$-tuple $\left(f_{1}, \cdots, f_{n}\right) \in A_{*}^{n}(M)$, i.e. $E$ is an affine $n$-boundary of $M$. Hence $E \supset E_{n}(M)$ because the latter is the smallest closed affine $n$-boundary of $M$.

Q.E.D. 
COROLlary 2. Let $\left\|\left(f_{1}, \cdots, f_{n}\right)(x)\right\|$ be one of the following convex functions:

$$
\sum_{j=1}^{n}\left|f_{j}(x)\right| ; \quad \max _{j=1}^{n}\left|f_{j}(x)\right| ; \quad\left(\sum_{j=1}^{n}\left|f_{j}(x)\right|^{p}\right)^{1 / p}, \quad p \geqq 2 .
$$

Then $E_{n}(M)$ is the smallest closed subset $E$ of $M$ that satisfies one of the following equivalent conditions:

1) $\min _{x \in E}\|F(x)\| \leqq \min \{\|x\|: X \in b F(M)\}$ for every $F=\left(f_{1}, \cdots, f_{n}\right) \epsilon$ $A^{n}(M)$;

2) $B(\min \{\|X\|: X \in F(E) \cap b F(M)\})$ is contained either entirely in $F(M)$ or entirely outside $F(M)$ for every $F \in A^{n}(M)$, where $B(r)$ is the open ball in $\boldsymbol{R}^{n}$ centered at the origin and with radius $r$;

3) $\min _{x \in E}\|F(x)\|=\min _{x \in M}\|F(x)\|$ for every regular $n$-tuple $F \in A_{*}^{n}(M)$;

4) $F$ vanishes within $E$ for every $F \in A^{n}(M)$ such that $b F(M) \ni$ $(0, \cdots, 0)$;

5) $B\left(\min _{x \in E}\|F(x)\|\right) \subset B(\min \{\|X\|: X \in F(E) \cap b F(M)\}) \subset F(M)$ for every $F \in A^{n}(M) \backslash A_{*}^{n}(M)$.

Proof. Actually every one of these conditions characterizes affine $n$-boundaries of $M$, as we shall see. 1 ) If $E$ is an affine $n$-boundary of $M$, then according to Theorem $2 F(E) \supset b F(M)$ for any $F \in A^{n}(M)$ and hence $\min _{x \in E}\|F(x)\|=\min _{X \in F(E)}\|X\| \leqq \min _{X \in b F(M)}\|X\|$. Conversely, if $E$ is not an affine $n$-boundary of $M$, then according to Theorem 2 there will exist an $n$-tuple $F=\left(f_{1}, \cdots, f_{n}\right) \in A^{n}(M)$ so that $F(E) \not \supset b F(M)$. If $X^{0} \in b F(M) \backslash F(E)$ and $x_{0} \in F^{-1}\left(X^{0}\right)$, then for the $n$-tuple $H=\left(f_{1}-x_{1}^{0}, \cdots, f_{n}-x_{n}^{0}\right) \in A^{n}(M)$ we have: $H\left(x_{0}\right)=(0, \cdots, 0) \in b H(M) \backslash H(E)$ since the set $H(M)$ can be obtained from $F(M)$ by a translation with $X^{0}$ and it preserves the topological properties of $\boldsymbol{R}^{n}$. Hence $0=\min _{X \in b H(M)}\|X\|<\min _{x \in E}\|H(x)\|$, i.e. condition 1) is not satisfied for the $n$-tuple $H \in A^{n}(M)$. 2) If $F \in A_{*}^{n}(M)$ then $\rho(O, b F(M))=$ $\rho(O, F(M))$ and hence $B\left(\rho(O, b F(M)) \subset \boldsymbol{R}^{n} \backslash F(M)\right.$ where $O=(0, \cdots, 0)$ and $\rho(O, N)=\inf _{X \in N}\|X\|$ is the distance in $\boldsymbol{R}^{n}$ from $O$ to the set $N \subset M$ with respect to the metric $\rho(x, y)=\|x-y\|$. Condition 1 ) now says that $B(\rho(O, F(E))) \subset B(\rho(O, b F(M))) \subset \boldsymbol{R}^{n} \backslash F(M)$. If $F \in A^{n}(M) \backslash A_{*}^{n}(M)$ then $\rho(O, b F(M))=\rho\left(O, \boldsymbol{R}^{n} \backslash F(M)\right)$ and hence $B(\rho(O, b F(M))) \subset F(M)$. Now 1) says that $B(\rho(O, F(E))) \subset B(\rho(O, b F(M))) \subset F(M)$, which proves the case 2). Because 1) implies 3$), 4$ ) and 5) for the corresponding $n$-tuples $F \in A^{n}(M)$, these conditions are fulfilled for every affine $n$-boundary $E$ of $M$. If $E$ is not an affine boundary then, as we saw above, condition 1) does not hold for the $n$-tuple $H$ with $(0, \cdots, 0) \in b H(M)$. This completes the proof of cases 4) and 5). By a suitable translation with some point $Y^{0} \in \boldsymbol{R}^{n} \backslash H(M)$ we can obtain also a regular $n$-tuple $H-Y^{0} \in A_{*}^{n}(M)$ such that $\rho(O, b(H-$ 
$\left.\left.Y^{0}\right)(M)\right)<\rho\left(O,\left(H-Y^{0}\right)(E)\right)$, i.e. $\min _{x \in E}\left\|H(x)-Y^{0}\right\|>\min _{\left(H-Y^{0}\right)(M)}\|X\|$ in contradiction with condition 3$)$.

Q.E.D.

The next corollary gives local characterizations of the points of the minimal affine $n$-boundary $E_{n}(M)$.

CoRollary 3. A point $x_{0} \in M$ belongs to $E_{n}(M)$ iff for any neighborhood $U$ of $x_{0}$ there exists an n-tuple $F \in A^{n}(M)$, such that:

1) $F \in A_{*}^{n}(M)$ and $\min _{U}\|F(x)\|<\min _{M \backslash U}\|F(x)\|$;

2) $b F(M) \ni(0, \cdots, 0)$ and $\min _{M \backslash U}\|F(x)\|>0$;

3) $F \in A^{n}(M) \backslash A_{*}^{n}(M)$ and $\rho(0, F(U) \cap b F(M))<\rho(0, F(M \backslash U) \cap b F(M))$.

Proof. If some of these properties fails to be true, then according to Theorem 1 or Corollary $2 E_{n}(M) \subset M \backslash U$ in contradiction with $x_{0} \in U$. If some of these properties holds for every $U \ni x_{0}$ this will imply that $U \cap E_{n}(M) \neq \varnothing$ so that every neighborhood of $x_{0}$ will contain points from $E_{n}(M)$, wherefrom $x_{0} \in E_{n}(M)$ since $E_{n}(M)$ is closed.

Q.E.D.

\section{§3. Some applications.}

The following is an affine version of classical Rouche's theorem for analytic functions and of its generalization for $n$-tuples of uniform algebra elements, due to Corach and Maestripieri, as well [5].

TheOREm 3. Let $M$ be a compact convex subset of $V$, and $F$ and $G$ be $n$-tuples from $A^{n}(M)$. If the inequality

$$
\|F(x)-G(x)\|<\|F(x)+G(x)\|
$$

holds on $E_{n}(M)$ then $F$ and $G$ are simultaneously regular or irregular $n$-tuples of $A^{n}(M)$.

Proof. Because the minimal affine $n$-boundary $E_{n}(M)$ is a compact subset of $M$, there will exist an integer $m$ such that:

$$
m \min _{E_{n}(M)}(\|F(x)+G(x)\|-\|F(x)-G(x)\|)>\max _{M}\|F(x)-G(x)\| .
$$

Assume that the theorem is not true. Then the end members of the sequence $2 m F,(2 m-1) F+G,(2 m-2) F+2 G, \cdots, F+(2 m-1) G, 2 m G$ are not simultaneously regular $n$-tuples over $A(M)$. Hence there are two neighboring members of this sequence, one of which is regular and the other is irregular. Suppose that $k$ is an integer such that the $n$-tuple $(m-k) F+(m+k) G$ is regular but $(m-k+1) F+(m+k-1) G$ is an irregular $n$ tuple and let $x_{0}$ be a point of $M$ for which $(m-k+1) F\left(x_{0}\right)+(m+k-1) G\left(x_{0}\right)=0$. Now 


$$
\begin{aligned}
\max _{x \in M} & \|F(x)-G(x)\|<m \min _{E_{n}(M)}(\|F(x)+G(x)\|-\|F(x)-G(x)\|) \\
& \leqq \min _{E_{n}^{(M)}}(m\|F(x)+G(x)\|-k\|F(x)-G(x)\|) \leqq \min _{E_{n}(M)}\|(m-k) F(x)+(m+k) G(x)\| \\
& =\min _{M}\|(m-k) F(x)+(m+k) G(x)\| \leqq\left\|(m-k) F\left(x_{0}\right)+(m+k) G\left(x_{0}\right)\right\| \\
& =\left\|(m-k) F\left(x_{0}\right)+(m+k) G\left(x_{0}\right)-\left[(m-k+1) F\left(x_{0}\right)+(m+k-1) G\left(x_{0}\right)\right]\right\| \\
& =\left\|G\left(x_{0}\right)-F\left(x_{0}\right)\right\| \leqq \max _{x \in M}\|F(x)-G(x)\| .
\end{aligned}
$$

The obtained contradiction proves the theorem.

Q.E.D.

An other application of minimal affine $n$-boundaries is the proving of the following affine version of a theorem of Hartogs for analytic functions in the unit ball in $\boldsymbol{C}^{n}$ and its generalization for pairs of uniform algebra elements, due to Sibony [6], as well.

THEOREM 4. Let $M$ be a compact convex subset of $V$, and $F$ and $G$ be $n$-tuples of elements of $A(M)$. If the equality

$$
\|F(x)\|=\|G(x)\|
$$

holds on $E_{2 n}(M)$ then it holds everywhere in $M$.

It is interesting to know if the minimal affine $n$-boundary $E_{n}(M)$ coincides with the closure of these end subsets of $M$, that are contained in $(n-1)$-dimensional affine subspaces of $V$ as in the case $n=1$.

Note. Recently M. Hayashi has proved positively this problem (private communication).

Acknowledgement. Thanks are due to the Department of Mathematics of Hokkaido University, where this paper was written, for its hospitality.

\section{References}

[1] K. Yosida, Functional Analysis, Springer-Verlag, 1965.

[2] R. Phelps, Lectures on Choquet's Theorem, Van Nostrand Math. Studies, 1966.

[3] T. TONEv, Minimal boundaries of commutative Banach algebras containing the Shilov boundary, Complex Analysis and Applications, Sofia, 1986, 712-714.

[4] T. TONEv, New relations between Sibony-Basener boundaries, Special Year in Complex Analysis, Univ. of Maryland, 1987, Lecture Notes in Math., Springer, 1277 (1987), 256-262.

[5] G. Corach and A. MAEstripieri, Expansions of characters and generalized Shilov boundaries, Revista de la Union Matematica, vol. 32, Nr. 3 (1987), 211-216.

[6] N. Sibony, Multidimensional analytic structure in the spectrum of a uniform algebra, Spaces of Analytic Functions, Kristiansand, Norway, 1975, Lecture Notes in Math., Springer, 512 (1976), 139-175.

Present Address:

Institute of Mathematics, Bulgarian ACademy of Sciences

BG-1090 Sofia, BUlgaria 